Polymer Journal Vol. 1, No. 3, pp. 296-303 (1970)

\title{
Orientation Behavior of Polyethylene Crystals
}

\author{
Toru TAKAHASHI and Ichiro SATO \\ Research Department, Fujimori Kogyo Company Ltd. \\ 56, Imai Kami-cho, Kawasaki, Japan.
}

(Received September 9, 1969)

\begin{abstract}
The orientation behavior of crystalline phases in the process of uniaxial stretching of tubular extruded polyethylene film with a hot-air type industrial stretcher is described quantitatively in terms of the degree of biaxial orientation and X-ray pole figure.

In the initial stretching stage, the $a$-axes are separated rather definitely into those oriented parallel to the $Z$-axis and those oriented perpendicular to the $Z$-axis. The degree of $\overline{\cos ^{2} \Phi_{z}}$ of $a$-axes and $c$-axes is same for both axes. The changes of $\overline{\cos ^{2} \Phi_{b, i}}$ which have taken place in the interval are negligible. From these phenomena it seems fairly justifiable to conclude that the greatest factor of the orientation mechanism in the initial stage is simple (100) [001] plastic slipping and rotation of the slip on a (100) plane around the $b$-axis. This structure, as the limiting case in the initial stretching process, is a combination of two simple structures in terms of crystal: the type I ( $a$-axis model) suggested by Holmes et al., and the fiber type. In terms of orientation functions of three principal crystallographic axes, a transformation from the Kobayashi's model or the type I model to the Fiber type model follows the rule of the type II model suggested by Keller.

KEY WORDS Polyethylene / Uniaxial Stretching / Orientation / X-

Ray / Pole Figure / Biaxial Orientation Function / Fiber Type / Transformation /
\end{abstract}

The orientation behavior of polyethylene crystallites in the process of uniaxial stretching of tubular extruded polyethylene films by a hot-air type industrial stretcher was examined.

The film deformation with uniaxial stretching was accompanied by a necking phenomenon during the high-speed fabrication of uniaxial stretched tapes in the $80-130^{\circ} \mathrm{C}$ range of temperatures.

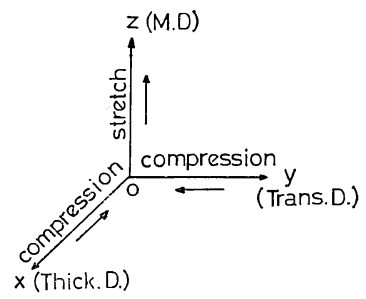

Figure 1. The film (Origin 0 ) is exposed to complicated force when stretched toward $Z$.

As shown in Figure 1, when stretched towards $Z$, a film is subject to compressions from $X$ and $Y$. These compressions are not balanced and the origin 0 is exposed to complicated force.

It is recognized, therefore, that a biaxial orienta- tion is required when the orientation behavior of crystallites under these conditions is considered.

In the present paper, the orientation mechanism of polyethylene crystallites in the system will be discussed quantitatively at first in terms of the $\mathrm{X}$-ray pole figures, ${ }^{1,2}$ and second, in terms of the degrees of the biaxial orientation function of the crystalline structural unit.

\section{EXPERIMENTAL}

\section{Test Specimens}

Test specimens were sampled from polyethylene film at various positions of the machine direction while they were in the deformation steps of the stretching process. Polyethylene films were continuously stretched through a $30 \mathrm{~cm}$-high and 300 $\mathrm{cm}$-long conventional hot air stretching bath at $120^{\circ} \mathrm{C}$. Two sets of nip roll were placed, one at the inlet of the stretching bath, the other at the outlet of the bath. Test specimens were left as they were for two hours after the continuously operated film-introducing and film-stretching nip rolls had been simultaneously and completely stopped and were chilled gradually. Polyethylene 
films between the nip rolls at both ends were taken off and sampled from each middle area in a transverse directions as shown in Figure 2.

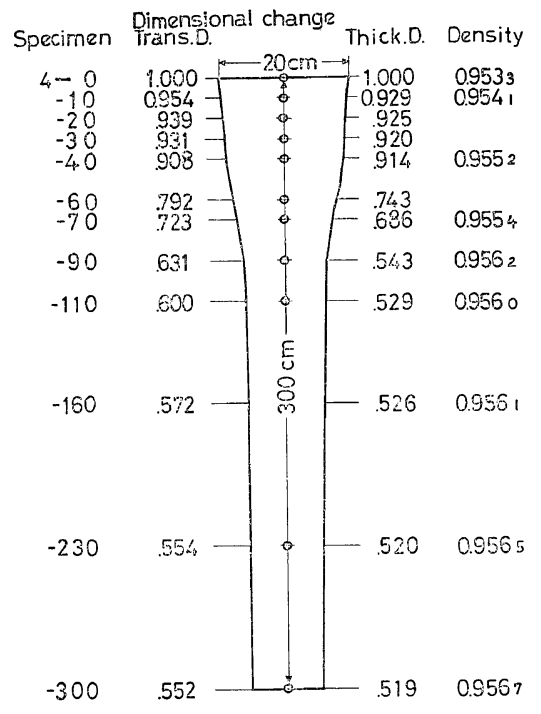

Figure 2. Explanation of the test specimens in the $4 X$-stretching process.

The film introducing speed of the stretching bath was $5 \mathrm{~m} / \mathrm{min}$ and the revolution ratio of the stretching nip roll to the introducing roll, known as a stretch ratio, was kept constant at 4 throughout the experiments described in this paper.

Figure 2 shows dimensional- and densitychanges of each specimen owing to the stretching action.

The film used was tubular extruded high density polyethylene - Hizex $5000 S$ (Mitsui Petrochemical Co., Ltd.) - with a $70 \mu$ thickness and a $20 \mathrm{~cm}$ width.

Evaluation of Biaxial Orientation of Principal Crystallographic Axes of Polyethylene Crystallites

Wilchinsky ${ }^{3}$ has expressed the average orientation of the $c$-axis in a reference direction $Z$, fixed in the sample, as the average square cosine $\overline{\cos ^{2} \Phi}$ of the angle between $c$ and $Z$. In a unit sphere, the angle between the normal $O N$ to a set of $h k l$ planes and the specific two axes $O Z$ and $O Y$ in a space to be measured are taken as $\Phi$ and $\psi$, respectively ( $c f$. Figure 3 ). The distribution density function of reciprocal lattice vectors of $h k l$ planes of crystallites on the surface

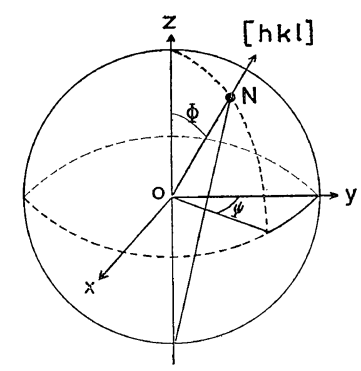

Figure 3. Position of plane normal $N$ located by spherical coordinates $\phi$ and $\phi$.

of a unit sphere is $N_{(\Phi, \psi)}$, where $N_{(\Phi, \psi)}$ is the pole concentration that expresses the relative amount of crystallites having plane normals in the $\Phi$ and $\psi$ direction. Considering $N_{(\varphi, \psi)}$, the average degree of biaxial orientation of the reciprocal lattice vectors can be obtained from the following set of two quantities:

$\overline{\cos ^{2} \Phi}=\frac{\int_{0}^{\pi / 2}\left[\int_{0}^{\pi} N_{(\Phi, \psi)} d \psi\right]_{\Phi} \cos ^{2} \Phi \sin \Phi d \Phi}{\int_{0}^{\pi / 2}\left[\int_{0}^{\pi} N_{(\Phi, \psi)} d \psi\right]_{\Phi} \sin \Phi d \Phi}$

$\overline{\cos ^{2} \psi}=\frac{\int_{0}^{\pi}\left[\int_{0}^{\pi / 2} N_{(\Phi, \psi)} \sin \Phi d \Phi\right]_{\Phi} \cos ^{2} \psi d \psi}{\int_{0}^{\pi}\left[\int_{0}^{\pi / 2} N_{(\Phi, \psi)} \sin \Phi d \Phi\right]_{\Phi} d \psi}$

Desper and Stein $^{4}$ have stated that a set of three $\overline{\cos }^{2} \Phi_{i}(i ; x, y, z)$ can be used instead of $\overline{\cos ^{2} \Phi}$ and $\overline{\cos ^{2} \phi}$ as shown in Figure 4. In order to find the value of $\overline{\cos ^{2} \phi_{i}}$ from the pole figure of $X$-center in Figure 5, Eqs. 3, 4, and 5 were used:

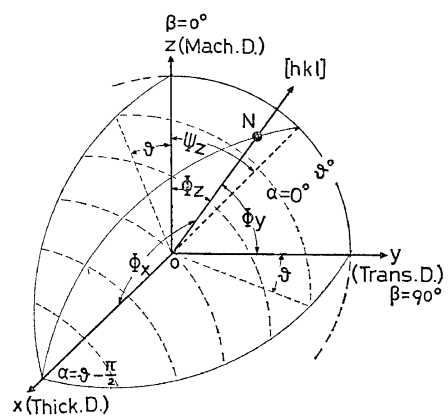

Figure 4. Orientation of the $[h k l]$ normal for the crystallites and location of $\Phi_{x}, \Phi_{y}, \Phi_{z}$. 


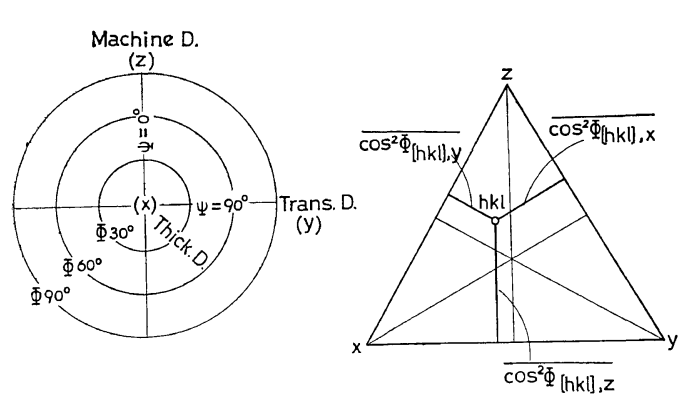

Figure 5. Location of the $[h k l]$ orientation function in the equilateral triangle plot (right) and location of the polar angle $\Phi_{x}$ and the azimuthal angle $\phi_{z}$ in the pole figure (left).

$$
\overline{\cos ^{2} \Phi_{x}}=\frac{\int_{0}^{\pi / 2}\left[\int_{0}^{\pi} N_{\left(\Phi_{x}, \psi_{z}\right)} d \phi_{z}\right]_{\Phi_{x}} \cos ^{2} \Phi_{x} \sin \Phi_{x} d \Phi_{x}}{\int_{0}^{\pi / 2}\left[\int_{0}^{\pi} N_{\left(\Phi_{x}, \psi_{z}\right)} d \phi_{z}\right]_{\Phi_{x}} \sin \Phi_{x} d \Phi_{x}}
$$$$
\overline{\cos ^{2} \Phi_{z}}=\frac{\int_{0}^{\pi / 2} \int_{0}^{\pi} N_{\left(\Phi_{x}, \psi_{z}\right)} \sin ^{3} \Phi_{x} \cos ^{2} \phi_{z} d \phi_{z} d \Phi_{x}}{\int_{0}^{\pi / 2} \int_{0}^{\pi} N_{\left(\Phi_{x}, \phi_{z}\right)} \sin \Phi_{x} d \phi_{z} d \Phi_{x}}
$$$$
{\overline{\cos ^{2} \Phi_{x}}}+{\overline{\cos ^{2} \Phi_{y}}}+{\overline{\cos ^{2} \Phi_{z}}}_{z}=1
$$

The present paper relates to the investigations involving the relationships in Figure 4 and Eqs. 3,4 , and 5 .

The distribution $N_{\left(\Phi_{x}, \psi_{z}\right)}$ at a particular polar angle $\Phi_{x}$ may be directly related to the X-ray diffraction intensity distribution as follows

$$
N_{\left(\Phi_{x}, \psi_{z}\right) \Phi_{x}} d \psi_{z}=K_{(\alpha, \beta)_{x}} d \beta
$$

where $K$ is a proportional constant, and $I_{(\alpha, \beta)}$ is an azimuthal distribution of X-ray diffraction intensity as measured by an ordinary horizontal scanning type diffractometer. The scanning angle used was twice the Bragg angle $2 \theta$ for reflection from the $h k l$ planes while keeping the tilting angle of the film normal ( $X$-axis) to the incidence of X-ray beams at a particular angle and rotating the film around the $X$-axis by angle $\beta$ from $0^{\circ}$ to $\pi$.

The measurement of $N_{\left(\Phi_{x}, \psi_{z}\right)}$ was then taken by setting a pole figure attachment on an X-ray goniometer and employing $\mathrm{Ni}$-filtered $\mathrm{Cu}-\mathrm{K}_{\alpha}$ rays. Each datum was corrected in respect of the background, absorption by amorphous regions, scattering, and overlapping of the adjacent peaks.

Orientation distribution of the crystallographic axes $a$ and $b$ was obtained by employing diffraction peaks of (200) and (020) planes. That of the crystallographic axis $c$ was obtained from an Eq. 7:

$$
\overline{\cos ^{2} \Phi_{a, i}}+\overline{\cos ^{2} \Phi_{b, i}}+\overline{\cos ^{2} \Phi_{c, i}}=1
$$

If the orientation is distributed randomly, then

$$
\overline{\cos ^{2} \Phi_{x}}=\overline{\cos ^{2} \Phi_{y}}=\overline{\cos ^{2} \Phi_{z}}=\frac{1}{3}
$$

If the orientation is completely parallel to the $Z$-axis, then

$$
\overline{\cos ^{2} \Phi_{z}}=1.0, \overline{\cos ^{2} \Phi_{y}}=\overline{\cos ^{2} \Phi_{x}}=0
$$

If the orientation is completely parallel to the $X$-axis, then

\begin{tabular}{|c|c|c|c|c|c|c|c|c|c|}
\hline \multirow{2}{*}{ Specimen } & \multicolumn{3}{|c|}{$\overline{\cos ^{2} \Phi_{a, i}}$} & \multicolumn{3}{|c|}{$\overline{\cos ^{2} \Phi_{b, i}}$} & \multicolumn{3}{|c|}{$\overline{\cos ^{2} \Phi_{c, i}}$} \\
\hline & $x$ & $y$ & $z$ & $x$ & $y$ & $z$ & $x$ & $y$ & $z$ \\
\hline $4-\quad 0$ & 0.304 & 0.227 & 0.469 & 0.445 & 0.517 & 0.038 & 0.251 & 0.256 & 0.493 \\
\hline $4-10$ & 0.386 & 0.306 & 0.308 & 0.449 & 0.510 & 0.041 & 0.165 & 0.184 & 0.651 \\
\hline 4- 20 & 0.394 & 0.303 & 0.303 & 0.452 & 0.502 & 0.046 & 0.154 & 0.165 & 0.651 \\
\hline $4-30$ & 0.404 & 0.318 & 0.278 & 0.420 & 0.533 & 0.047 & 0.176 & 0.149 & 0.675 \\
\hline $4-40$ & 0.461 & 0.319 & 0.220 & 0.421 & 0.526 & 0.053 & 0.118 & 0.155 & 0.727 \\
\hline $4-60$ & 0.452 & 0.376 & 0.172 & 0.418 & 0.519 & 0.063 & 0.130 & 0.105 & 0.765 \\
\hline $4-70$ & 0.483 & 0.408 & 0.109 & 0.422 & 0.513 & 0.065 & 0.095 & 0.079 & 0.826 \\
\hline 4- 90 & 0.518 & 0.431 & 0.051 & 0.422 & 0.521 & 0.057 & 0.060 & 0.048 & 0.892 \\
\hline $4-110$ & 0.506 & 0.450 & 0.044 & 0.426 & 0.526 & 0.048 & 0.068 & 0.024 & 0.904 \\
\hline $4-160$ & 0.514 & 0.442 & 0.044 & 0.441 & 0.527 & 0.032 & 0.045 & 0.031 & 0.924 \\
\hline $4-230$ & 0.519 & 0.452 & 0.029 & 0.437 & 0.531 & 0.032 & 0.044 & 0.017 & 0.939 \\
\hline
\end{tabular}

$$
\overline{\cos ^{2} \Phi_{x}}=1.0, \overline{\cos ^{2} \Phi_{y}}=\overline{\cos ^{2} 0 \Phi_{z}}=0
$$

Therefore, the value of $\overline{\cos ^{2} \Phi_{i}}$ varies in the range 0 to 1.0 .

Table I. Biaxial orientation of three crystallographic axes of polyethylene crystallites 


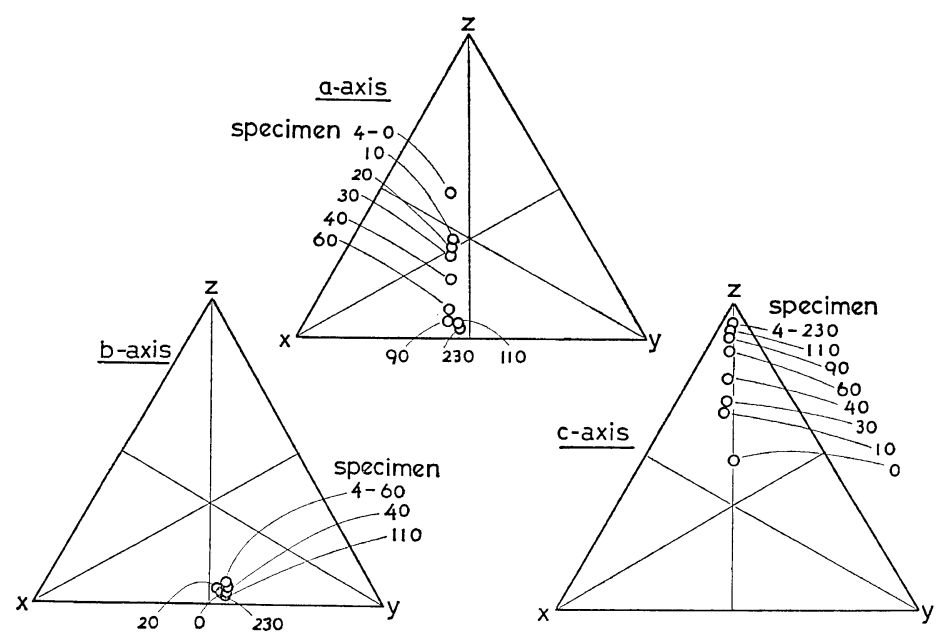

Figure 6. Orientation function diagrams for a change of $\overline{\cos ^{2} \Phi_{[h k l], i}}$ in the process of stretching.

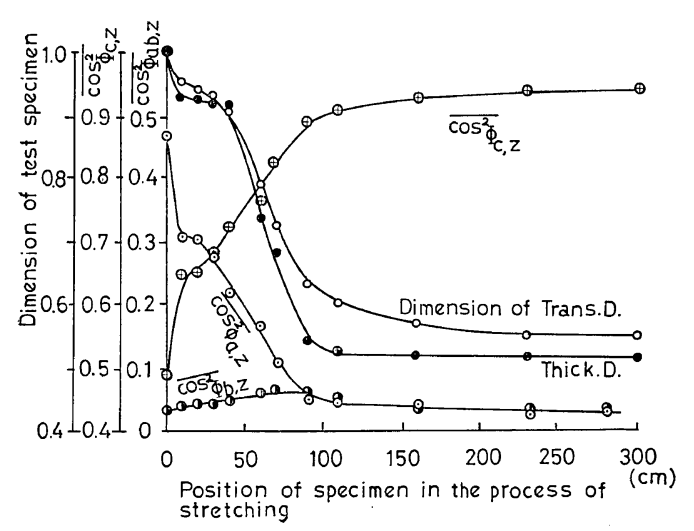

Figure 7. $\overline{\cos ^{2} \Phi_{[h k l], z}}$ and dimensional changes of polyethylene films in the process of stretching.

\section{EXPERIMENTAL RESULTS AND DISCUSSION}

The biaxial orientation functions of polyethylene films in each position of the machine direction under stretching are listed in Table I, are shown in Figure 7 and are shown in the $X Y Z$ triangular plot in Figure 6. The pole figures obtained for these samples are shown in Figures 8-1 to 8-7.

Specimen 3-0 is considered as still unstretched, the $b$-axis lies perpendicular to the screw axis, the $a$ - and $c$-axes are tilted at various angles to the $Z$-axis, and the two angles are complementary to each other. In terms of orientation function this specimen is a parallel point in a model proposed by Kobayashi. ${ }^{5}$ The average orientation angle of $a$-axes of crystallites is found from an Eq. 8.

$$
\Phi_{x}=\frac{\int_{0}^{\pi / 2}\left[\int_{0}^{\pi} N_{\left(\Phi_{x}, \psi_{z}\right)} d \psi_{z}\right]_{\Phi_{z}} \Phi_{x} \sin \Phi_{x} d \Phi_{x}}{\int_{0}^{\pi / 2}\left[\int_{0}^{\pi} N_{\left(\Phi_{x}, \psi_{z}\right)} d \psi_{z}\right]_{\Phi_{x}} \sin \Phi_{x} d \Phi_{x}}
$$

Then the average orientation angle of the $a$-axis against the $X$-axis is $60.24^{\circ}$.

In the initial stretching stage, (100) planes
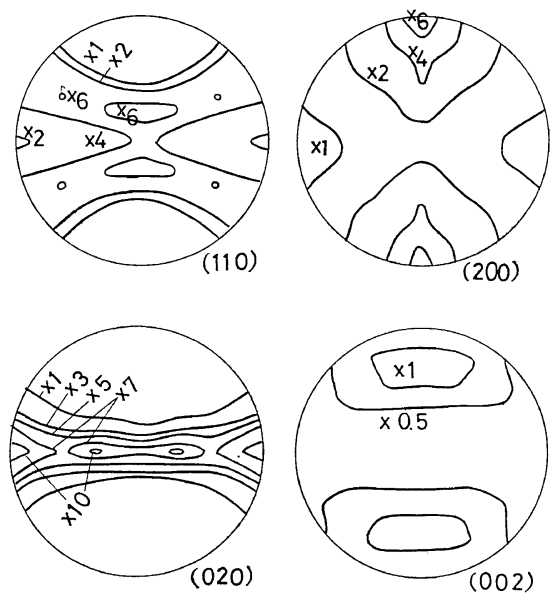

SPECIMEN 4-0

Figure 8-1. Pole figures of polyethylene film in the process of stretching. 

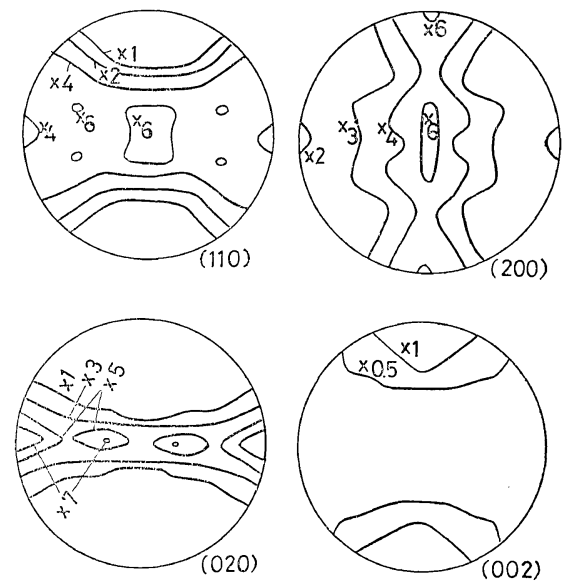

$-4-10$

Figure 8-2. (Continued).
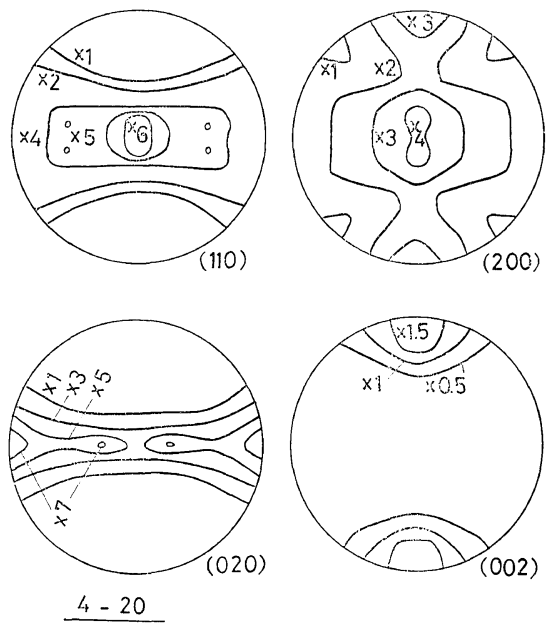

Figure 8-3. (Continued).

become active at elevated temperatures of film and plastic deformations of crystal occur by slipping. The direction of the slip is the direction [001]. (100) [001] plastic slipping and rotation of the slip on a (100) plane around the $b$-axis do not proceed simultaneously at a slow rate in all the crystals, but they occur at first in those crystals oriented in such a manner that the $a$-axis angle of inclination with the $Z$-axis is greater. They gradually propagate other crystals so oriented that the $a$-axis angle together with the $Z$-axis becomes smaller. Once the [100] axis has begun to rotate around the $b$-axis, the displacement of the [100] axis proceeds at quite a
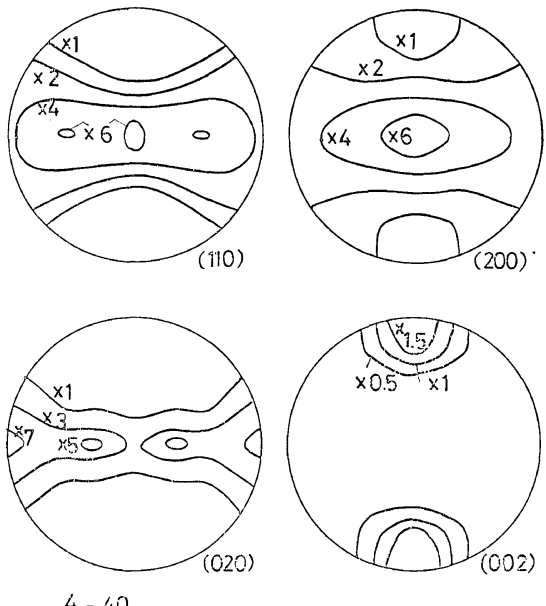

Figure 8-4. (Continued).
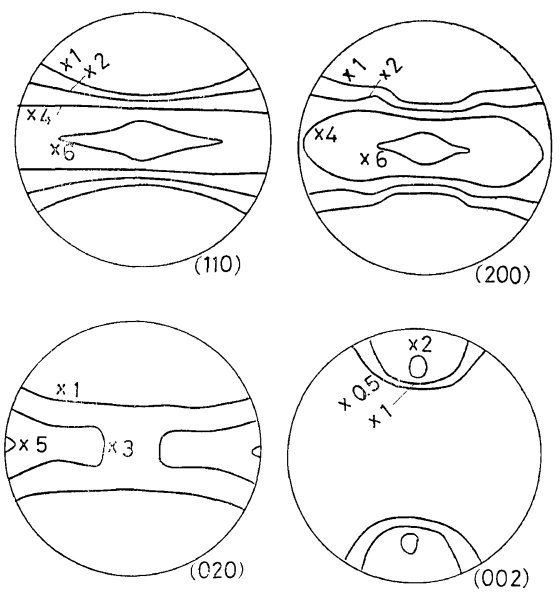

$4-70$

Figure 8-5. (Continued).

good rate. The pole figures in Figures 8-2, 8-3 and 8-4 show that the [200] axes are separated rather definitely into those oriented parallel to the $Z$-axis and those oriented perpendicular to the $Z$-axis. In specimen $4-20$, the value of $\overline{\cos ^{2} \Phi_{a, z}}$ approaches that of the random orientation but is due to the coexistence of the crystallites whose $a$-axes are highly oriented, perpendicular and parallel to the $X$-axis as listed in Table II. In terms of crystal orientation this structure is a combination of two simple structures, the fiber type (c-oriented towards the fiber axis $Z$, with $a$ and $b$ random about $c$ ) and the Type I ( $a$-axis model) suggested by Holmes et al. ${ }^{8}$ The dimen- 


\section{Orientation Behavior of Polyethylene Crystals}
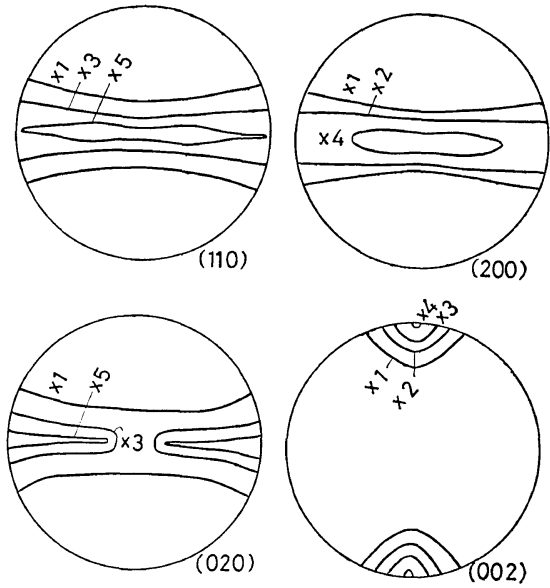

$4-90$

Figure 8-6. (Continued).
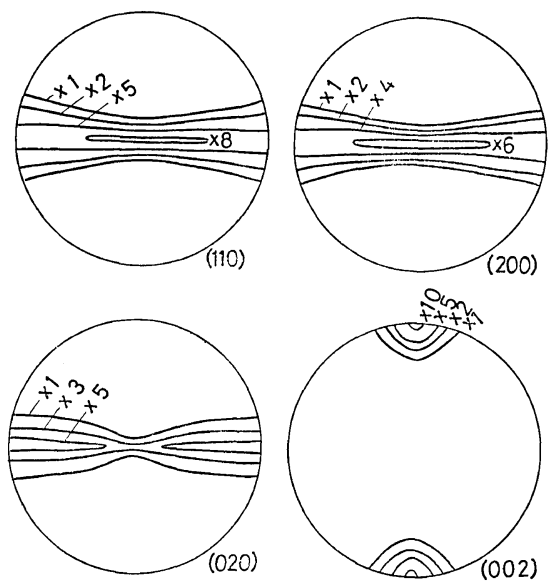

$\underline{4-230}$

Figure 8-7. (Continued).

Table II. Degree of the average biaxial orientation function of crystal $a$-axes in the test specimen 4-20 after separation into [200] $]_{\|}$and $[200]_{\perp}$ ingredients in the chart

\begin{tabular}{ccc}
\hline & $\begin{array}{c}{[200]_{\|}} \\
\text {(Parallel to } \\
\text { the } X \text {-axis) }\end{array}$ & $\begin{array}{c}{[200]_{\perp}} \\
\text { (Perpendicular to } \\
\text { the } X \text {-axis) }\end{array}$ \\
\hline$\overline{\cos ^{2} \Phi_{a, x}}$ & 0.514 & 0.259 \\
$\frac{\cos ^{2} \Phi_{a, y}}{\cos ^{2} \Phi_{a, z}}$ & 0.384 & 0.183 \\
\hline
\end{tabular}

sional changes during the stretching process are most remarkable in the initial stage corresponding to the remarkable change of the $\overline{\cos ^{2} \Phi_{z}}$ of

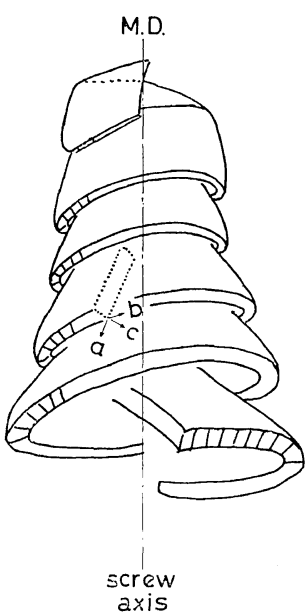

Figure 9. Schematic diagram of a lamellar texture in Specimen 4-0. The original idea has been proposed by Kobayashi et al. (refs. 5 and 6).

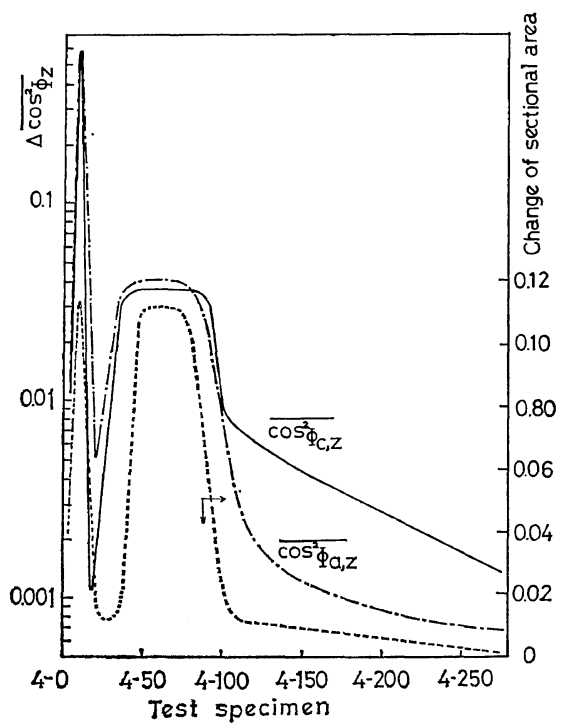

Figure 10, Change in quantity of $\overline{\cos ^{2} \Phi_{a, z}}, \overline{\cos ^{2} \Phi_{c, z}}$ and sectional area at an interval of $10 \mathrm{~cm}$ for machine direction in the process of stretching.

the $a$ and $c$ axes. These phenomena can be seen in Figures 7 and 10. The changes of $\overline{\cos ^{2} \Phi_{b, i}}$ which have taken place in the interval are negligible. When rotation increases the resolved shear stress on a second slip or an unfolding of lamellar crystals appears to give a value sufficient to make it also become active, but experimental results are positive in denying the 
fact. As the changes of $\overline{\cos ^{2} \Phi_{z}}$ of $a$ - and $c$-axes correspond exceedingly to each other, in the 4-0 to 4-90 positions, it seems fairly justifiable to conclude that the greatest factor of the orientation mechanism in the initial stage is the simple (100) [001] plastic slipping and rotation of the slip on a (100) plane around the $b$-axis.

As the stretching process goes on, the (100) [001] slip and the rotation of the slip on a (100) plane around the $b$-axis propagate gradually until they cover the whole of the crystallites. This is observed to occur at the position $4-50$ to $4-60$ of the specimen in Figure 2.

From a position 4-100 on, dimensional changes become negligible, and the value of $\overline{\cos ^{2} \Phi_{c, z}}$ is recognized to increase gradually while the value $\overline{\cos ^{2} \Phi_{a, z}}$ decreases slightly. This phenomenon may be attributable to the same degrees of contribution of the changes of $\overline{\cos ^{2} \Phi_{b, i}}$ and $\overline{\cos \Phi_{a, z}}$ to the change of $\overline{\cos ^{2} \Phi_{c, z}}$. One of the parts of this phenomenon may be the fact that adjacent intermolecular bonds are melted during heating in the stretching process. New crystallization in the folded form and the orientation functions during the stretching process of the crystallites that existed prior to this process will not closely coincide with those of the recrystallized crystallites formed in the stretching process at this time. The formation of the recrystallized crystallites during the stretching process is presumed by an increase in the density and the acknowledgement that the (002) diffraction intensity at $\Phi_{x}=90^{\circ}$ and $\phi_{z}=0^{\circ}$ becomes greater as the stretching process approaches the final stage in spite of the fact that the smaller shifts of $\overline{\cos ^{2} \Phi_{c, i}}$ are seen. The (002) diffraction intensity at $\Phi_{x}=90^{\circ}$, $\phi_{z}=0^{\circ}$ and the average orientation angle $\Phi_{x}$ of the crystal $c$-axis obtained directly, using the (002) reflection are shown in Figure 11. The comparison between the orientation functions of the $c$-axis obtained directly, using the (002) reflection and that calculated from the orientation functions of (200) and (020) reflections is listed in Table III. It is found that these two values coincide with each other. The results of Figure 11 seem to suggest that the orientation of crystallites recrystallized in the stretching process have grown with their molecular axes completely united with the stretching axis ( $Z$-axis).

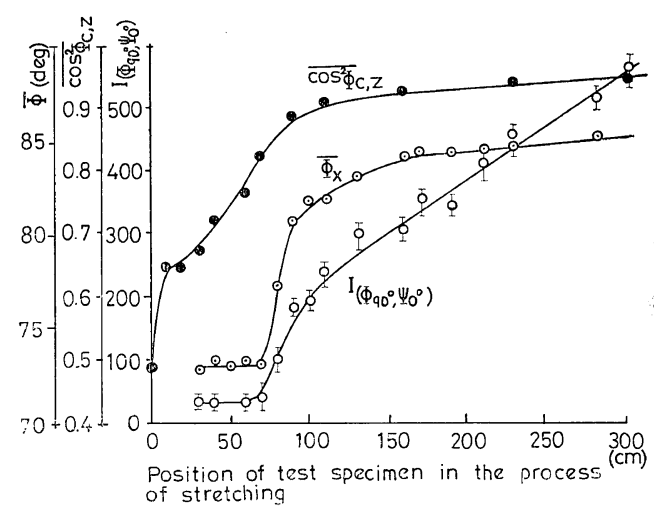

Figure 11, Average orientation angle $\left(\overline{\Phi_{x}}\right)$ of $c$-axis, $\overline{\cos ^{2} \overline{\Phi_{c, z}}}$ and (002) diffraction intensity $(2 \theta=$ about $\left.74.60^{\circ}\right)$ at $\Phi=90^{\circ}, \phi=0^{\circ}$ of test specimens.

Table III. The comparison between the orientation functions of the crystal $c$-axis obtained directly, using (002) reflection and that calculated from the orientation functions of (200) and (020) reflections

\begin{tabular}{ccc}
\hline & \multicolumn{2}{c}{$\overline{\cos ^{2} \Phi_{c, z}}$} \\
\cline { 2 - 3 } & $\begin{array}{c}\text { Calculated from } \\
\frac{\cos ^{2} \Phi_{a, z} \text { and }}{\cos ^{2} \Phi_{b, z}}\end{array}$ & $\begin{array}{c}\text { Obtained directly, } \\
\text { using (002) } \\
\text { reflection }\end{array}$ \\
\hline $4-90$ & 0.892 & 0.896 \\
$4-110$ & 0.904 & 0.908 \\
$4-160$ & 0.924 & 0.923 \\
$4-230$ & 0.939 & 0.944 \\
\hline
\end{tabular}

Following deformation processes of polyethylene in the stretching of the film are interpreted in terms of crystal orientation:

(1) The greatest factor of the orientation mechanism in the initial stage is the simple (100) [001] plastic slip and rotation of the slip on a (100) plane around the $b$-axis. And the initial order of the orientation distribution of the $b$-axis about the screw axis in the matrix is still kept up at the initial stretching stage without being affected by the (100) [001] slip deformation or a recrystallization. The experimental results seem to suggest that a transformation from the Kobayashi's model or the type I model to the Fiber model follows the rule of the type II model suggested by $\operatorname{Keller}^{9}$ ( $b$ orients perpendicular to $Z$, with $a$ and $c$ rotating about $b$ ). 
(2) As the stretching approaches the final stage in spite of the fact that the smaller shifts of the orientation function of the $c$-axis are seen, an oriented crystallization will then take place in the the folded form. And the orientation of the crystallites recrystallized in the stretching process have grown with the molecular axis completely united with the stretching axis.

Acknowledgment. The authors are indebted to Prof. Hiromichi Kawai of Kyoto University, Dr. Hirokazu Takahara of Bridge-Stone Tire Co., Ltd. and Dr. Takashi Oda of Nagoya Institute of Technology, for their valuable discussions and kind guidance on biaxial orientation functions. Thanks are also due to Prof. Seizo Okamura of Kyoto University, for his advice and encouragement.

\section{REFERENCES}

1. B. F. Decker, E. T. Asp, and Harker, J. Appl. Phys., 19, 388 (1948).

2. L. G. Schulz, J. Appl. Phys., 20, 1033 (1949).

3. Z. W. Wilchinsky, J. Appl. Phys., 31, 1969 (1960).

4. C. R. Desper and R. S. Stein, J. Appl. Phys., 37, 3990 (1966).

5. K. Kobayashi in "Polymer Single Crystals", P. H. Geil, Ed., Interscience, New York, 1963, p. 465-475.

6. K. Kobayashi and T. Nagasawa, J. Polym. Sci, Part C, 15, 163 (1966).

7. K. Kobayashi, The 26th Annual Meeting of the Research Institute for Chemical Fibers, Japan Oct., 1968.

8. D. R. Holmes, R. G. Miller, R. P. Palmer, and C. W. Bunn, Nature, 171, 1104 (1953).

9. A. Keller, Nature, 174, 926 (1954). 